https://helda.helsinki.fi

\title{
Gefühl und Erfahrung
}

\section{Lindén, Jan-Ivar}

Wilhelm Fink verlag

2021

pÿLindén , J-I 2021, Gefühl und Erfahrung . in G Agostini \& H Christin (eds), Pathos

Affektformationen in Kunst, Literatur und Philosophie : Festschrift zu Ehren von Gerhard

Poppenberg . Wilhelm Fink verlag , Paderborn , pp. 567-585 . https://doi.org/10.30965/9783846765579_027

http://hdl.handle.net/10138/340438

https://doi.org/10.30965/9783846765579_027

cc_by

publishedVersion

Downloaded from Helda, University of Helsinki institutional repository.

This is an electronic reprint of the original article.

This reprint may differ from the original in pagination and typographic detail.

Please cite the original version. 


\title{
Gefühl und Erfahrung
}

\author{
Jan-Ivar Lindén
}

\section{Präliminarien}

Im Folgenden werde ich den Begriff der Welt so verwenden, wie wir es in der Geschichte tun, wenn wir von der Welt der Römer oder von der minoischen Welt sprechen, oder, etwa in der Ethologie, wenn wir die Ameisenwelt oder die Welt der Löwen meinen. Den Begriff der Wirklichkeit verwende ich für dasjenige, was die Welt als besonderen Bedeutungsraum trägt - was gleichzeitig bedeutet, dass die Welt einen Zugang zur Wirklichkeit darstellt. Welt wird also als eine besondere Bestimmung der Wirklichkeit verstanden. Wer an einer solchen Bestimmung teilhat, lebt in der entsprechenden Welt. Wenn die Zugehörigkeit zu einer Welt hervorgehoben wird, impliziert dies also nicht, dass Welten voneinander gänzlich getrennte Seinsbereiche wären. Ganz im Gegenteil liegt die Wirklichkeit der Welten weitgehend darin, dass Überschneidungen vorkommen, z. B., wenn sich Löwen und Menschen begegnen. Die Vielfalt der Welten impliziert die Existenz der Alterität in jeder einzelnen Welt. Alterität gibt es jedoch nicht nur 1) durch andere Welten, die aus der Sicht einer besonderen Welt zwar als Bestandteil der eigenen Welt bestimmt sein können, aber zugleich unbestimmt sind, insofern sie für Fremderfahrung stehen, ${ }^{1}$ sondern auch 2) als das schlechthin Unbestimmte, das keine entfliehende Fremderfahrung andeutet. Alterität kann also sowohl für Andersweltlichkeit wie auch für Weltverschlossenheit stehen. Ontologisch sind die beiden Formen der Alterität wesentlich.

Die Weltverschlossenheit soll nicht ihrer Unbestimmtheit wegen mit Materialität verwechselt werden. Gerade die Materie ist in der Tradition jedoch als eine unbestimmte Basis verstanden worden, die durch (In-)Formierung Gestalt annehmen kann. Diese nicht nur unter Aristotelikern verbreitete Sichtweise hat eine relative Berechtigung, insofern wir von Dingen in unserer Welt sprechen. Die Materie kann aber nie als basale Wirklichkeit verstanden werden, weil die Gestaltung von etwas Unbestimmtem eine besondere Beziehung darstellt, in der etwas innerhalb einer gewissen Welt seine Form offenbart. Es ist deshalb ausgeschlossen, z. B. den Gegenstandsbereich einer spezifischen, instrumentell bedingten Experimentalwissenschaft als die materielle Wirklichkeit darzustellen. Solche Gegenstände sind von ihren

1 Wie wird der Löwe mich wahrnehmen? 
Erkenntnisbedingungen besonders abhängig und gerade die Bedingungen sind ontologisch zentral, nicht die Gegenstände. Für die Gegenstände der Experimentalwissenschaft ist weitgehend kennzeichnend, dass sie gerade keine Fremderfahrung andeuten und deshalb als innerweltliche Phänomene auf radikale Weise Andersweltlichkeit ausklammern. Dies gilt aber nicht nur für die Experimentalwissenschaft und damit verbundene Konzeptionen der Materialität. Vergegenständlichung ist generell unser Mittel, uns auch dann orientieren zu können, wenn das Andere keine Stimme hat und auch sonst keinen Zugang zu ihm als Fremderfahrung erlaubt. Martin Buber hat dies als ein $E s$ verstanden, das nur durch Verdinglichung handzuhaben ist. ${ }^{2}$ Objektivierung wäre in diesem Licht dasjenige, was uns übrig bleibt, wenn sich der Bereich der reziproken Erfahrung verschließt und wir deshalb vor allem auf unsere aktive Fähigkeit, etwas möglichst genau zu bestimmen, angewiesen sind. In der Objektivierung geht es folglich um eine Bestimmungsinitiative. Dies scheint auch auf die intentionale Konstitution von Gegenständen zuzutreffen, insbesondere wenn die Konstitutionsleistung im Geiste Kants transzendentalphilosophisch verstanden wird. Gibt es andere Möglichkeiten, den Begriff des Intentionalen zu verstehen?

\section{Die Wirklichkeit der Welt}

Was in Welten wirkt, ist nicht von unserer Fähigkeit zur Gegenstandskonstitution abhängig. Vielmehr besitzt es als Anlass die Initiative und konkretisiert sich, indem wir in der Erfahrung mitwirken - und dabei auch gegenständliche Konturen zu erfassen vermögen. Eine Welt kann sogar als dasjenige verstanden werden, was Mitwirkung in der Wirklichkeit ermöglicht. Kein epistemologisch oder transzendentalphilosophisch gesteigerter Subjektivitätsbegriff kann diese unhintergehbare Zugehörigkeit zum Wirklichen aufheben, und die Erfahrung ist, so gesehen, ein Medium, in dem sich Wirklichkeit ausdrückt. Dieser Bereich umfasst weit mehr als nur konstituierte oder konstituierbare intentionale Gehalte - auch dann, wenn diese Gehalte nicht reduktionistisch auf die besonders kontrollierte und sogar teilweise hergestellte Erfahrung durch Experimente zurückgeführt werden. Empirisch im eigentlichen Sinn ist diejenige Ontologie, die dieser fundamentalen Dimension Rechnung zu tragen vermag. In diesem Licht muss der ontologische Stellenwert der Affektivität verstanden werden und dies bedeutet zugleich, dass es eine gewisse Spannung zwischen Intentionalität und Affektivität gibt.

2 Martin Buber, Ich und Du, Stuttgart: Reclam, 1995 [1923]. 
Einer einseitigen Hervorhebung der Konstitutionsleistung muss entgegengehalten werden, dass Erfahrung grundsätzlich von demjenigen abhängt, was nicht Anhalt durch intentionalen Gehalt bietet, sondern vielmehr den Anlass der Erfahrung darstellt und folglich die Bestimmungsinitiative des Erfahrenden relativiert. Auch die intentionale Bestimmung ist veranlasst, und die ontologische Herausforderung ist es, zu entscheiden, was über das Veranlassende gesagt werden kann - allem voran, ob es im Anlass neben Unbestimmtheit auch nicht-konstituierte Bestimmtheit gibt, und wie darüber sinnvoll gesprochen werden kann.

Wer subjektphilosophisch die Bestimmungsinitiative zu einem Bestimmungsmonopol überhöht, wird behaupten müssen, dass der Anlass der Erfahrung als Anlass unbestimmt ist, und dass folglich jede Bestimmtheit der Erfahrung aus einer bestimmenden Aktivität (oder Spontaneität) seitens des Subjekts herrührt. Wenn Subjektivität dann auch anthropomorph verstanden wird, führt dies zu einem unbefriedigenden Anthropozentrismus. Zugang zu gewinnen, ist legitim, aber was sich in oder durch einen Zugang zeigt, kann nie mit dem gleichgesetzt werden, wozu der Zugang ein Zugang ist.

Dies bedeutet, dass innerweltliche Bestimmtheit ihren ontologischen Grund in etwas viel Unbestimmterem hat, aber nicht, weil der Zugang zum An sichSeiendem ganz einfach verschlossen wäre (Kant), sondern weil der Erfahrende selbst auch von Unbestimmtheit durchzogen ist, insofern er wirklich ist. Diese Wirklichkeit drückt sich als Betroffenheit aus. Was einen betrifft, ist weder in einer Außen- noch einer Innenwelt zu lokalisieren: in keiner Innenwelt, weil es um Wirklichkeit geht und in keiner Außenwelt, weil diese Wirklichkeit zwar gegeben ist, aber nicht als äußeres sinnliches Material, nicht als „das Mannigfaltige der Anschauung“ (Kant), ${ }^{3}$ auch nicht als der sinnliche Einflussbereich einer Außenwelt (Condillac) ${ }^{4}$ oder überhaupt als etwas spatial Situiertes. Die immer noch gängige Auffassung der sinnlichen Erfahrung als einer besonderen Handhabe von äußerlich gegebenen sinnlichen Daten kann deshalb nie dem Anlass der Erfahrung Rechnung tragen. ${ }^{5}$ Gewiss gibt es in der Erfahrung etwas,

3 Immanuel Kant, Kritik der reinen Vernunft, z. B. B 139.

4 Étienne Bonnot de Condillac, Traité des sensations, Paris: Fayard, 1984 [1754].

5 Die Annahme von Sinnesdaten ist in der Wahrnehmungstheorie häufig kritisiert worden. Eine Quelle ist Ludwig Wittgenstein, Philosophische Untersuchungen, Frankfurt a. M.: Suhrkamp, 1980 [1953]. In seiner Darstellung der interpretativen Wahrnehmung (anhand der sog. Hasenente) hat Wittgenstein Anstöße der Gestaltpsychologie philosophisch weiterentwickelt. Einige Literaturangaben zur Diskussion über Sinnesdaten finden sich in Perceiving, Sensing and Knowing. Hg. von Robert J. Swartz, New York: Doubleday, 1965. Als typischer Vertreter der Annahme von Sinnesdaten kann Alfred Ayer dienen, z. B. in: ders., The Foundations of Empirical Knowledge, London: Macmillan, 1963 [1940]. 
was auf Respons pocht, und das Wort ,Datum‘ besagt schon rein sprachlich, dass etwas gegeben ist. Was genau gegeben ist, bleibt aber ein philosophisches Problem. Gegeben ist etwas für denjenigen, der vom Gegebenen betroffen ist. Diese Betroffenheit ist zwar eine Art Ausgesetztheit, aber um sich aussetzen zu können, muss der Erfahrende zugleich etwas tun oder jedenfalls ausdrücken. Kant hat deshalb ganz folgerichtig Rezeptivität mit Spontaneität verknüpft. Die Frage ist nur, wie dieses Begriffspaar letztendlich verstanden werden muss.

\section{Realitätssinn}

Dass es in der Erfahrung Responsivität und Reaktivität gibt, ist offensichtlich, und verschiedene Modi dieser Empirie sind uns vertraut: Einige werden mit den Sinnesvermögen verbunden, andere mit persönlichen Gefühlen, die entweder momentane Affekte oder andauernde Emotionen sein können und die in gewissen Fällen auch Stimmungen genannt werden. Eine Stimmung kann häufig eine Gesamtsituation prägen und ist deshalb nicht zwangsläufig an eine Person gebunden. Dazu kommt, dass es paradoxerweise häufig gerade für die Stimmung kennzeichnend ist, unbestimmt zu sein. Lässt sich daraus vielleicht ein Hinweis entnehmen, warum Erfahrung so eng mit Unbestimmtheit verbunden ist? Ist die Stimmung vielleicht gerade die ontologisch tragende Dimension der Affektivität? ${ }^{6}$

6 Otto Bollnow unterscheidet zwischen intentional gerichteten Gefühlen und intentional unbestimmten Stimmungen und verwendet dabei das aus Heideggers Sein und Zeit stammende Beispiel von Furcht und Angst. Wir fürchten etwas Bestimmtes, aber wovor wir Angst haben, bleibt dagegen unbestimmt - und ist gerade seiner Unbestimmtheit wegen angsterregend, unheimlich. Im Gefühl der Freude freuen wir uns analog auf etwas, wogegen Fröhlichkeit eher eine Stimmung ohne intentionales Korrelat ist (Otto Bollnow, Das Wesen der Stimmungen, Würzburg: Königshausen \& Neumann, 2009 [1941], S. 22). Bollnow muss jedoch selbst bemerken, dass die Trennung von Gefühl und Stimmung häufig schwierig ist. Eine gewisse Unbestimmtheit scheint tatsächlich für die Emotionalität als solche zu gelten, auch dann, wenn sie etwas Bestimmtes im Blickfeld hat. Wenn im Titel dieses Beitrags von Gefühl gesprochen wird, ist der Begriff umfassend zu verstehen. Sowohl momentane Affekte wie auch andauernde Emotionen werden als Gefühle verstanden. Der Begriff der Stimmung drückt wiederum aus, dass Gefühle die Erfahrung tragen und hebt zugleich hervor, dass die Affektivität keinen psychologischen Innenbereich darstellt, sondern jenen Bedeutungsraum bezeichnet, der unterschiedliche affektive und andere Positionierungen ermöglicht. Heidegger nennt dies die „Angänglichkeit“ des Daseins (Martin Heidegger, Sein und Zeit, Tübingen: Max Niemeyer Verlag, 1986 [1927], S. 137 und generell § 29). Gemeint ist ein Grundzug des In-der-Welt-seins, das als Relationsexistenzial immer schon bedeutsam ist. Nach der Darstellung in Sein und Zeit ist die „Befindlichkeit“ das „Da“ schlechthin, wozu das Dasein sich auf unterschiedliche Weise verhält und zwar, nach Heidegger, zumeist „in 
Wenn Gefühle besondere Ausdrücke tragender Stimmungen sind, die grundsätzlich aus einer vor der Unterscheidung zwischen Subjekt und Objekt liegenden und äußerst wirklichen Einbezogenheit in der Welt stammen, ergibt sich die Möglichkeit, auch die sinnliche Wahrnehmung in diesem affektiven Kontext einzuordnen. Die Wahrnehmung wird dann nicht als Erfassen äußerer Daten, sondern als zunehmende Bestimmtheit im Affektiven verstanden. Wie dieser Vorgang erscheint, hängt allerdings mit der Inkarnation des Lebewesens und insofern auch mit den jeweils gegebenen Sinnesvermögen zusammen. Wahrzunehmen heißt, so verstanden, sich durch Einordnung und Erfassen besser orientieren zu können. ${ }^{7}$

Die sinnlichen Qualitäten, die sich z. B. als Laut und Licht ausdrücken, setzen eine Wirklichkeit voraus, die vor der bewegungsabhängigen Unterscheidung zwischen Innen- und Außenwelt liegt. In dieser Wirklichkeit ist Einiges offensichtlich dazu geeignet, sich objektivieren $\mathrm{zu}$ lassen und vom Gefühlsbereich der Wirklichkeit loszulösen, während Anderes sich gerade in sinnlicher Empfindung, Gefühl und Emotion manifestiert. Diese letztere affektive Dimension sollte nicht vorschnell als weniger wirklich beschrieben werden. Es ist jedoch denkbar, dass die Affektion vor allem dann orientierungsbefördernd ist, wenn sie sich sinnlich differenzieren lässt und

der Weise der ausweichenden Abkehr" (Sein und Zeit, S. 136). Diese Perspektive zeigt einen Unterschied zwischen Aristoteles und Heidegger. Während bei Heidegger die ausweichende Abkehr, die mit Uneigentlichkeit verknüpft ist und deshalb als Gegenkraft Entschlossenheit einfordert, im Zentrum steht, ist bei Aristoteles nicht die Abkehr, sondern vielmehr die Zuwendung das Tragende in der beseelten Existenz, die von Streben und teleologischer Richtung geprägt ist. Die Unterschiede zwischen Heidegger und Aristoteles betreffen auch die theoretische Haltung, die in Sein und Zeit als ein pures Betrachten des Vorhandenen beschrieben wird (Sein und Zeit, §§ 15-18) und deshalb keine ontologisch tragende Funktion haben kann. Zugleich hebt Heidegger jedoch hervor, dass keine theoretische Einstellung der Befindlichkeit entkommen kann. Später wird von der gedämpften theoretischen Stimmung als einer Art Langeweile gesprochen (Martin Heidegger, Die Grundbegriffe der Metaphysik. Welt - Endlichkeit - Einsamkeit, Frankfurt a. M.: Klostermann, 1983 [Vorlesungen 1929-30]). Aus aristotelischer Sicht ist das theorein eher mit eudaimonia verbunden, d. h. mit einer lustbetonten Stimmung, in der die Welt nicht als unheimlich, sondern als verwunderlich, vielleicht dann und wann als abstoßend, aber häufig auch als schön erscheint. Es stimmt aber auch, dass die theoria nach Aristoteles eher eine Auszeichnung oder Vervollkommnung der menschlichen Existenz darstellt, keine ursprünglich gegebene Faktizität. Die faktisch gegebene Natur ist bei Aristoteles von animalischen Lebensbedingungen stark geprägt und auch in der aristotelischen Wahrnehmungstheorie stellt die Ausgesetztheit des Lebewesens seine natürliche Grundbedingung dar. In dieser Hinsicht könnte auch bei Aristoteles von einer „Betroffenheit“ des Daseins bzw. des Lebewesens gesprochen werden. Die Frage ist, wie eine solche affektive Betroffenheit ontologisch verstanden werden soll und was sie für das Verhältnis zwischen Gefühl, Empfindung, Wahrnehmung und Erfahrung bedeutet.

7 Man vergleiche das krinein bei Aristoteles. 
mit den leiblichen Sinnesorganen entsprechend verbunden ist. Zugleich gilt aber, dass es ohne Gefühle der Sinnlichkeit an Reizbarkeit fehlen würde. Um Missverständnissen vorzubeugen, ist es hier wichtig, die Idee einer subjektiven Konstitution der Gegenstände fernzuhalten. Sinnesorgane und emotionale Dispositionen sind - ebenso wie die Wirklichkeit generell - von einer langen Vorgeschichte geprägt, und was in der Erfahrung erscheint, ist von dieser vorherbestimmenden Geschichte massiv beeinflusst. Vorherbestimmende Wirklichkeit in einen subjektiven und einen objektiven Bereich aufzuteilen, ist kaum sinnvoll.

Die relative Berechtigung einer Grenzziehung zwischen Innen- und Außenwelt hängt mit unserer Beweglichkeit zusammen. Dazu gehört die Trennung zwischen dem eigenen, spontan zugänglichen und beweglichen Leib einerseits und der Umgebung andererseits. Diese Trennung ist zweifellos für ein sich um Orientierung bemühendes und von Orientierung abhängiges Lebewesen wesentlich. Auf dieser Ebene gibt es deshalb durchaus etwas Äußeres, das in der Orientierung räumlichen Anhalt bieten kann - entwicklungsgeschichtlich zuerst durch Tastsinn und Geschmack, dann auch durch die Fernsinne. Auf dieser Ebene kann von einem Zusammenhang zwischen Widerständigkeit und Gegenständlichkeit gesprochen werden. Man muss zugleich in Erinnerung halten, dass es nur dann Widerständigkeit und die sich daraus ergebende Lernfähigkeit geben kann, wenn die erfahrende Instanz durch ihre Leiblichkeit selbst Räumlichkeit enthält - aber dann eben nicht als widerständigen Raum, sondern als ein sich in der Welt einrichtendes, Platz suchendes und Abstände einschätzendes Wesen.

In der Gegenwart wird etwas aktualisiert und zwar in besonderen Situationen. Wenn die Aktualisierung bedeutsam ist, sprechen wir von Erfahrung. Erfahrung ist, so gesehen, ein Sonderfall der Wirklichkeit, aber aus unserer Sicht ein besonders wichtiger Fall, weil wir ohne Erfahrung kaum etwas fühlen, verstehen oder erkennen würden. Wie sich Wirklichkeit in unserer Erfahrung aktualisiert, verspricht uns deshalb etwas über Aktualisierung, Dispositionalität, Präsenz, Sinnlichkeit, Affektivität, Wahrnehmung, Erkenntnis und Verständnis zu verdeutlichen. Als Erfahrung ist die Wirklichkeit eben besonders vertraut. Empirischen Belang von sogenannten Sinnesdaten abzuleiten und den Wirklichkeitsbezug auf diese Weise einzuengen, wäre eine Verkürzung.

Wenn Aktualisierung erfahren wird, bezieht sie auch Gefühle ein. Es scheint immer noch der Vorteil des Erlebnis-Begriffs zu sein, dass er der Affektivität auf sprachlich einleuchtende Weise Rechnung trägt. Das Leben erreicht im Erlebnis eine spürbare Intensitätsstufe. Unter Berücksichtigung der Tatsache, dass Leben einen Begriff für nicht-inerte Natur darstellt, wären Erlebnisse deshalb 
Kristallisationspunkte der bedeutsam gewordenen Natur, die auf diese Weise zu reizbarer Erfahrung wird. Dies würde andeuten, dass emotionale Spontaneität nicht so sehr eine subjektive Funktion darstellt, sondern vielmehr für die Aktualität einer besonderen Beziehung steht, derer der Erfahrende gerade dann teilhaftig ist, wenn er sich einem möglichen Einfluss aussetzt. Diese Teilhaftigkeit erlaubt Stufen, die als Stufen der Intensität verstanden werden können. Was wirkt, ist in diesem Sinn wirklich - aber dass etwas wirklich ist, heißt nicht, dass es deshalb auch verstanden oder erkannt wird. Die Wirklichkeit der Gemütsregungen implizieren weder eine erkennende noch verstehende Aufnahme des Wirklichen ins Bewusstsein. Was würde sich aber epistemisch und hermeneutisch ergeben, wenn erfahrene Wirklichkeit vor allem stimmungshaft ist? Der Anlass der Erfahrung wäre dann jedenfalls kein äußeres Material für mentale Verarbeitung, sondern vielmehr so angelegt, dass er vor der Unterscheidung zwischen Innerem und Äußerem liegt.

\section{Wesen im Unbestimmten}

Insofern Unbestimmtheit sowohl als absolute als auch als relative Unbestimmtheit verstanden werden kann, gibt es hier ein Problem. Absolut unbestimmt ist, was keinerlei Bestimmtheit besitzt. Um relativ unbestimmt zu sein, genügt es dagegen, dass etwas für eine erfahrende Instanz unbestimmt bleibt. Was relativ unbestimmt ist, kann, absolut gesehen, durchaus eine gewisse Bestimmtheit besitzen. Wenn wir z. B. annehmen, dass es wirkliche Gesetzmäßigkeiten gibt, die auch dann vorliegen, wenn sie gar nicht erfahren werden, wäre eine Bestimmtheit gegeben, die mehr als nur relativ ist. Die platonische Tradition ist dafür bekannt, die Existenz einer absoluten Bestimmtheit vorauszusetzen, aber dieselbe Annahme ist auch - in unterschiedlicher Deklination - in anderen Denksystemen präsent. Bestimmtheit nur von menschlicher Erfahrung abzuleiten, scheint einen anthropologischen Reduktionismus zu implizieren, und deshalb ist es verständlich, wenn platonische und essentialistische Theorien sich verweigern, Bestimmtheit nur mit menschlicher Erfahrung zu verknüpfen. Diese Einsicht kann etwa folgendermaßen zusammengefasst werden: Alles, was für uns unbestimmt ist, muss nicht an sich unbestimmt sein. Es ließe sich aber hinzufügen - und dies ist ontologisch nicht weniger wichtig: Alles, was für uns bestimmt ist, muss nicht in derselben Weise an sich bestimmt sein. Deshalb kann z. B. die gegenständlich bestimmte Welt intentionaler Gehalte nicht als eine an sich gegebene Wirklichkeit verstanden werden. Trotz seiner subjektphilosophischen (kartesisch-kantischen) Neigung hat auch Husserl sein Prinzip der Epoché so ausgedrückt, dass es nicht um die Ausklammerung der 
Wirklichkeit ging, sondern nur um die Enthaltung des Urteils, dass intentionale Gehalte als solche wirklich sind.

Ontologisch verstanden, bedeutet Essentialismus, dass es in der Wirklichkeit wesentliche Züge gibt, die unabhängig davon gegeben sind, ob jemand sie erfährt oder nicht. Dies besitzt eine gewisse Plausibilität, weil sich damit erklären lässt, wie wir nachträglich verstehen können, dass etwas soeben Erkanntes schon vorher existierte. Was dieses Wesentliche ist, bleibt jedoch eine Herausforderung für die Philosophie. Die moderne Tendenz, Wesen mit Objekten zu vermengen, ist leider immer noch verbreitet (und dies nicht nur außerhalb der Philosophie). Was gegenständlich intendiert wird, hängt mit Orientierungswillen und Interessen zusammen und hat ontologisch gesehen seinen Grund in demjenigen, was solche Orientierungen und Interessen veranlasst, nicht in konstituierten Gehalten, die Anhalt in der Orientierung bieten. Ursprünglich ist der Anlass, nicht der Anhalt. In diesem Sinn wird im Folgenden die Frage nach dem Anlass der Erfahrung gestellt.

\section{Wie unbestimmt ist der Anlass der Erfahrung?}

Die erste Antwort wäre: Unbestimmt ist der Anlass der Erfahrung, insofern Erfahrung einen Vorgang darstellt, bei dem der Erfahrende durch Bestimmung Anhalt in seinen interessegeladenen Unternehmungen $\mathrm{zu}$ finden versucht und dabei als Anfangsdatum immer etwas relativ Unbestimmtes hat. Auf das Unbestimmte zu antworten, bedeutet in dieser Hinsicht, dem Unbestimmten durch aktives Bestimmen jedenfalls teilweise zu entkommen. Als Triebwesen ist diejenige erfahrende Instanz, die uns Sprechenden besonders vertraut ist - nämlich der Mensch - in seinem Tun und Lassen vom Unbestimmten getrieben. Diese Quelle des Trieblebens kann eben deshalb besonders spürbar sein, weil Unbestimmtheit keine bestimmbare, äußere Materialität darstellt, sondern vor der Unterscheidung in Innen- und Außenwelt in uns wirkt. Für unseren Bezug zu diesem Bereich wäre Teilhabe immer noch das adäquate Wort. Auch die Position der Angst bei Kierkegaard und Heidegger ist in diesem Licht zu verstehen. Besonders bei Heidegger gibt es jedoch die Tendenz, dieses Unbestimmte oder ,Unheimliche‘ als ein Gegenpol zu menschlichen Entwürfen zu verstehen. Dies führt zu einer starken Hervorhebung der ontologisch schöpferischen Rolle des menschlichen Daseins, und es ist deshalb kein Zufall, dass es in Sein und Zeit einen ontologischen Primat der (entworfenen) Zukunft gibt. Dazu gehört auch die Kunstauffassung von Heidegger, welche die besondere Geschichtlichkeit der Kunst als eine stiftende versteht. ${ }^{8}$ In Die

8 Martin Heidegger, Der Ursprung des Kunstwerkes, Stuttgart: Reclam, 1988 [1950], S. 79 . 
Grundbegriffe der Metaphysik drückt er diese Grundannahme wie folgt aus: Der Mensch ist weltbildend. ${ }^{9}$

Damit ist es begründet, einige Einsichten der essentialistischen Metaphysik wieder zu behaupten, um einer anthropologischen Reduktion der Bestimmtheit entgegenzuwirken. Nur muss dabei deutlich hervorgehoben werden, dass die Wesensdimension in der Befindlichkeit ihren Ausdruck findet. Wesentlich ist das, wovon wir besonders stark betroffen sind. So verstanden lässt die Wesensannahme durchaus - wie auch der Sprachgebrauch anzeigt - komparative Formen zu. Einiges ist wesentlicher, anderes weniger wesentlich - und das heißt eben, gut aristotelisch, beiläufig, akzidentell.

\section{Erfahrung und Erlebnis}

Erfahrung bedeutet, dass etwas in einem wahrnehmungsfähigen Lebewesen während eines Vorgangs prägend wirkt und zu künftigen Verhaltensdispositionen beiträgt. Dieses Geschehen kann reaktiv sein, aber auch responsiv, wenn der Erfahrende das, was ihm geschieht, gleichzeitig auch zu befragen vermag. In beiden Fällen ist das empirische Moment dasjenige, was den Unterschied zwischen reiner Selbstbezogenheit und Alteritätsoffenheit ausmacht. Deshalb hat der Begriff der Erfahrung auch in der Erkenntnis- und Wissenschaftstheorie eine zentrale Bedeutung gehabt. ,Empirische' Forschung ist, so verstanden, diejenige Tätigkeit, die Gedachtes mittels etwas Anderem als Denken zu überprüfen versucht. Es bleibt jedoch unklar, wie die bestätigende oder umwerfende empirische Instanz letztendlich zu verstehen ist. Erfahrung gibt es nicht nur in sogenannten Erfahrungswissenschaften, sondern vielmehr ist Erfahrung ein wesentliches Kennzeichen des Lebens. Typisch für den Begriff ist auch die komparative Form: mit der Zeit kann jemand erfahrener werden. Ein Begriff wie ,Erlebnis‘ kann dagegen schlecht komparativ gesteigert werden: Es klingt seltsam zu sagen, dass jemand mit der Zeit ,erlebter' wird. Dies deutet an, wie eng Erfahrung mit Lernfähigkeit verbunden ist. Zugleich entsteht jedoch die Frage, ob dieses orientierende Vermögen den Begriff der Erfahrung erschöpft. Vielleicht gibt es im momentanen Erleben etwas, das für die empeiria unumgänglich ist?10 Das Schlüsselwort in diesem Kontext ist Intensität - ein Aspekt, der als gefühlsmäßig verstanden werden

9 Martin Heidegger, Die Grundbegriffe der Metaphysik, § 42-46 und § 64-71. Beim späten Heidegger ändert sich allerdings diese Sichtweise - ein Thema, das hier ausgeklammert werden muss.

10 Vgl. den Begriff des Erlebnisses in der sogenannten Lebensphilosophie. Dilthey ist hier immer noch die einschlägige Quelle, aber vgl. auch die Ausführungen in Otto Bollnow, Lebensphilosophie und Existenzphilosophie, Würzburg: Königshausen \& Neumann, 2009. 
kann. Wenn Erfahrung sowohl mit Gefühlen wie auch mit Lernfähigkeit und Orientierungsvermögen verbunden ist, bietet sich ein Begriffspaar an, um diese innere Spannung der Erfahrung anzuzeigen: Intensität und Intentionalität. Die Intentionalität bedeutet Gerichtetsein, sei es als praktisch motivierte Absicht, erfassendes Denken (Meinen) oder zielgerichtetes Streben. Ohne jetzt zu dem Verhältnis zwischen diesen Formen der Intentionalität Stellung zu nehmen, ${ }^{11}$ kann vermutet werden, dass sie alle orientierungsbefördernd und deshalb eng mit Lernfähigkeit verbunden sind. Kann dasselbe von der affektiven Intensität gesagt werden? Die Intensität kann größer oder geringer sein, aber als solche scheint sie keine dispositionale Entwicklung zu bedeuten, wie es bei Lernvorgängen der Fall ist. Dennoch muss die Erfahrung eine Intensität besitzen, um zu künftigen Verhaltensdispositionen beitragen zu können. Ungefühlte Erfahrung scheint schwer vorstellbar zu sein.

\section{Gefühl, Stimmung, Empfindung}

Traditionell hat der Begriff der Affektion (affectio) eine Vielfalt von Gemütslagen gedeckt: andauernde Stimmungen wie Trauer, momentane Zustände wie der Schreck und auch Empfindungen wie Schmerz. Dazu wurden die Sinnesqualitäten (Licht, Ton usw.) als Affektionsqualitäten verstanden. Es ist deshalb schwierig, eine allgemeine Antwort auf die Frage zu geben, ob Affektion eine Lernfunktion ist oder nicht. Ein klassisches Beispiel, das eine enge Beziehung anzudeuten scheint, ist das Kind, das sich am Feuer verbrennt und so lernt, Feuer künftig zu vermeiden. Affektion wäre hier eine Lernfunktion. Es fragt sich jedoch, ob das Kind Feuer nicht als etwas Brennendes (intentional) meinen muss, um klüger zu werden. Wie die Debatte um sogenannte ,Sinnesdaten' (d. h. eine neutral gegebene Basis der Wahrnehmung) zeigt, ist es besonders schwierig zu sagen, was in der Empfindung auf keine Weise gemeint, d. h. als etwas Bestimmtes intendiert ist. Es scheint aber so etwas geben zu müssen, wenn die sinnliche Erfahrung für Alterität stehen soll. Dies ist in gewisser Hinsicht das Problem der Unbestimmtheit in der Wahrnehmung. In der Neuzeit, und auf besonders durchdachte Weise bei Kant, wird das Unbestimmte als unbestimmtes Material für subjektive Konstitutionsleistungen verstanden, das in einer nur denkbaren, aber empirisch nicht beweisbaren Verbindung mit den ,Dingen an sich' steht. Als Materie oder Material für Konstitutionsleistungen wird die Affektion zur Basis heruntergestuft und

11 Vgl. z. B. Jan-Ivar Lindén, „Intentionnalité et perception: une esquisse aristotélicienne“, in: Chôra (2011 / 2012). 
unterscheidet sich demgemäß von Gefühlen und Stimmungen, die umgekehrt eher eine uns überwältigende Macht besitzen. Diese emotionale Macht war in der älteren Tradition, allem voran in der Moralpsychologie und der Rhetorik, eine Selbstverständlichkeit - und die Ethik stellte weitgehend einen Versuch dar, mit solchen stark wirkenden Triebkräften zurechtzukommen. Zugleich gab es selbstverständlich auch die Annahme von sinnlichen Reizen, die ebenso mit Affektion verbunden wurden. Auf Deutsch ist der Begriff der Empfindung in signifikanter Weise zweideutig und steht einerseits für Gefühl, andererseits auch für sinnliche Empfindung in der Wahrnehmung. Wie unterscheidet sich dann ,die Macht der Gefühle‘ von sinnlichen Reizen? Die erste Antwort wäre zweifellos, dass Gefühle nicht zwangsläufig mit Einzelsinnen korrespondieren (obwohl Tasten und Fühlen sehr nah verwandt zu sein scheinen). Gehör, Gesichtssinn usw. beziehen sich anscheinend auf einen bestimmten Bereich möglicher Erfahrung: das Tönende, das visuell Erscheinende etc. Gefühle wie Glück, Heiterkeit und Furcht sind dagegen nicht, oder jedenfalls nicht direkt, mit Qualitäten einzelner Sinne verbunden. Trotzdem kann die spezifische Sinneserfahrung gefühlsmäßig konnotiert sein. Ist es überhaupt denkbar, dass sinnliche Reize wirken können, wenn das wahrnehmende ,Subjekt’ oder - um die Behandlung abgegrenzt zu halten - der wahrnehmende Mensch keine Gefühle besitzt? Wie tragen Gefühle und Stimmungen (kurz: das Emotionale) zur Wahrnehmung bei? Wie unterscheiden sich sinnliche Empfindungen von dem, was in ihnen emotional ist?

Eine Möglichkeit ist es, das Emotionale als die Grundbedingung der Reizbarkeit zu verstehen. Der emotive Zustand - sei er auch sachlich zurückgeschraubt, oder von Langeweile geprägt - macht das Wahrnehmbare bedeutsam für den Wahrnehmenden. Wenn die emotive Aufregung besonders groß ist, wird die Empfänglichkeit für Reize entsprechend stark. Ein gedämpfter emotiver Zustand erlaubt es dagegen, zielbewusst sich auf bestimmte Reize zu konzentrieren, was häufig Orientierung und bisweilen auch instrumentelle Geschicktheit begünstigt. ${ }^{22}$ Die Welt erscheint als ein Bereich von erfassbaren Dingen, bietet deutlichen Anhalt und zwar durch das, was wir in der Wahrnehmung als bestimmte Gehalte wahrnehmen. Dies ist ein besonders typischer Fall von Intentionalität, aber der Akt, durch den ein intentionaler Gehalt erfasst wird, ist als Akt von Aktualität geprägt. Aktualität kann mehr

12 Maine de Biran hat dieses Verhältnis zu analysieren versucht. Er unterscheidet in dieser Hinsicht die passive Empfindung (sensation) von der aktiv erfassenden Wahrnehmung (perception). Vgl. vor allem Maine de Biran, Influence de l'habitude sur la faculté de penser, in: ders., CEuvres. Hg. von François Azouvi, 13 Bde., Paris: Vrin, Bd. 2 (1987). Hg. von Gilbert Romeyer-Dherbey: Mémoires sur l'influence de l'habitude, S. 124-336. 
oder weniger Eigeninitiative enthalten, aber soll nicht mit spontaner Aktivität gleichgesetzt werden. Aktualität heißt vor allem Präsenz - und Präsenz ohne Affektivität zu denken, ist schwierig.

Wie sind dann die Reize als solche gegeben? Kann überhaupt sinnvoll von Reizen in der Welt gesprochen werden, wenn kein reizbares Wesen vorausgesetzt wird? Oder soll vielleicht mit Aristoteles davon ausgegangen werden, dass die Wirklichkeit tatsächlich eine qualitative Vielfalt darstellt, die eigenständig vorliegt, aber nur dann auch aktuell erfasst wird, wenn aus möglichen Reizen tatsächliche werden? Seit Aristoteles' Bemerkungen über aisthêta ist die Debatte nicht weit vorangekommen. Jedoch gab es im letzten Jahrhundert eine umfassende Diskussion, zuerst über die Möglichkeit von Sinnesdaten, dann über sogenannte qualia, d. h. was das gemeinsam Gemeinte stets überschreitet und nur persönlich empfunden werden kann (wie z. B. Zahnschmerz von vielen auch sehr genau beschrieben und bezeichnet werden kann, aber trotzdem nicht zum persönlichen Zahnschmerz wird). ${ }^{13}$ Ein Problem entsteht, weil es schwierig ist, Schmerz eindeutig als Inhalt oder Akt einzuordnen. Schmerz scheint kein intentionaler Gehalt zu sein, aber eben auch kein meinender Akt, vielmehr so etwas wie ein Modus, ein Wie des Meinens, die spezifische affektive Färbung des Meinens - was aus einem Meinen mein Meinen macht. ${ }^{14}$

\section{Meinung und Besinnung}

Um unsere Mitwirkung in der Wirklichkeit zu erhellen, gibt es unterschiedliche Strategien, die auch in der Wissenschaftstheorie erörtert worden sind, häufig durch die Unterscheidung von Erklären und Verstehen. Ich werde diese Diskussion hier nicht wieder aufgreifen, sondern versuche stattdessen, den Begriff der Intentionalität als Leitfaden zu nehmen, um eine Art der Seinserhellung von einer anderen, weniger beachteten Form zu unterscheiden. Die

13 Die Verteidigung von Qualia richtet sich häufig gegen einige Annahmen in Wittgensteins Philosophische Untersuchungen, die den Eindruck erwecken, es ginge Wittgenstein darum, sich gegen die philosophische Relevanz persönlicher Erlebnisse zu richten. Die Pointe in der sogenannten Analyse der Privatsprachen scheint jedoch eher diejenige zu sein, dass solche Erlebnisse nie die Bedeutung eines Wortes ausmachen können. Aus phänomenologischer Sicht ist jedoch auch diese Annahme problematisch. Vgl. Edmund Husserl, Erfahrung und Urteil. Untersuchungen zur Genealogie der Logik, Hamburg: Meiner, 1999.

14 Man vergleiche die zwei Möglichkeiten, sich darüber zu versichern, dass man tatsächlich wach ist und nicht nur träumt: Affektionsbezogen ist die Überprüfung, wenn man sich kneift. Anhaltsorientiert und intentional, dagegen, ist die Tendenz, besser zu fokussieren. 
zweite Form der Seinserhellung scheint mit Expressivität verbunden zu sein, insofern sie sich responsiv zu etwas verhält, was sich in uns durch Wirkung ausdrückt. Responsivität unterscheidet sich durch ein Wechselspiel von Frage und Antwort von Reaktivität und ist gerade deshalb hermeneutisch erhellend. Sie kann auch von der intentionalen Bestimmung verschiedener Erfahrungsgehalte profitieren, aber ist als solche mit keiner Konstitutionsleistung verbunden. Vielmehr wird in der responsiven Seinserhellung die eigene Existenz in Frage gestellt. Dies gelingt nur, wenn der Erfahrende sich persönlich aussetzt, aber zugleich die Fähigkeit beibehält, nicht nur erleidend Wirkendes aufzunehmen, sondern das Wirkende auch in reflexiver Verarbeitung zu vertiefen. Man könnte diese zwei Formen der Seinserhellung intentionale Konstitution bzw. reflexive Besinnung nennen. ${ }^{15}$

\section{Aristotelische Perspektiven}

Aristoteles beschreibt in De anima, wie der Wahrnehmungsvorgang darin besteht, etwas von dem Wahrgenommenen durch Assimilation zu retten. Das „Ungleiche“ wird „gleich“, d. h. in die Lebensrichtung (in die Entelechie) des Lebewesens integriert. ${ }^{16}$ Diese Assimilation hat ihren Hintergrund in der Tatsache, dass jedes Lebewesen in seinem Leben durch sein Lebensprinzip (psychê) Zwecke verwirklicht. Andere beseelte Wesen streben anders, und einige Dinge sind nach Aristoteles leblos. Erfahrung ist deshalb stets mit einem heteron verbunden. Mit dieser Andersheit zurechtzukommen, ist die Aufgabe der aisthêsis, und diese ist deshalb auch auf die eigene strebende Lebenswirklichkeit angewiesen. Wenn es bei Aristoteles intentionale Richtung gibt, ist sie psychisch und keineswegs ein Kriterium des Bewusstseins. Durch die Seele entsteht Streben (orexis), und wo Streben vorliegt, gibt es auch Erstrebtes (orekton). Intentionalität ist grundsätzlich mit Lebensvorgängen verbunden, und die explizit meinenden Formen der Intentionalität bleiben stets in diesen vitalen Kontext eingebettet. ${ }^{17}$ Innerhalb des Strebens muss der Strebende jedoch mit demjenigen rechnen, was nicht auf dieselbe Weise wie er selbst oder, im Falle lebloser Dinge, sogar überhaupt nicht wie Lebewesen strebt.

15 Man vergleiche den Begriff der Besinnung bei Dilthey, z. B. Wilhelm Dilthey, Grundlegung der Wissenschaften vom Menschen, der Gesellschaft und der Geschichte, in: ders., Gesammelte Schriften. Hg. von Helmut Johach und Frithjof Rodi, 26 Bde., Göttingen: Vandenhoeck \& Ruprecht, Bd. 19 (1982).

16 De anima $417 \mathrm{a}-\mathrm{b}$.

17 Vgl. Jan-Ivar Lindén (Hg.), Aristotle on Logic and Nature, Leuven: Peeters, 2019, insbesondere die Beiträge im Teil „Intentions and Directions“. 
Orexis ist mit aisthêsis verwoben, weil es dieses Andere zu berücksichtigen gilt. Für das strebende Lebewesen ist es wichtig, am Leben zu bleiben, und Aristoteles scheint die Erfahrung mit potentiell auch zerstörerischen Einflüssen zu verbinden. Übermäßige sensitive Intensität kann dazu führen, dass ein Sinnesorgan geschädigt wird, und in der taktilen Empfindung kann dies sogar bedeuten, dass das ganze Lebewesen zugrunde geht. ${ }^{18}$ Es ist deshalb nicht verwunderlich, dass das menschliche Leben emotional geladen ist. Erfahrung ist gefährlich und die Welt ist dem Wahrnehmenden auch deshalb wichtig, bedeutsam. ${ }^{19}$

In der Wahrnehmungstheorie kommen jedoch auch sinnliche Qualitäten wie Licht und Laut vor, und zwar so, dass der Wahrnehmende das Wahrgenommene (aisthêton) durch solche Qualitäten zu erfassen vermag. Diese Qualitäten tragen sozusagen die Form des Wahrgenommenen, die in der Wahrnehmung nach Aristoteles nicht mehr die Materialität besitzt, die sie in den Dingen selbst hatte. Die sinnlichen Qualitäten, die aisthêmata, erlauben dem Wahrgenommenen, unabhängig von seiner dinglichen Existenz, im Wahrnehmungsvorgang weiterzuwirken und fungieren dabei als Träger der wahrgenommenen Gestalten. Diese Vermittlung spielt sich in einem Medium (metaxu) ab. Zugleich ist jedoch auch eine gewisse Beständigkeit des in der Wahrnehmung entmaterialisierten Wahrgenommenen notwendig, weswegen die aisthêmata an Vorstellungen (phantasmata) gebunden sind, die im beweglichen Lebewesen erzeugt werden, um Einheit im Vorgang herzustellen. Licht und Laut können wechseln, aber ein identischer Vorstellungsgehalt kann trotzdem bestehen bleiben. Die Vorstellungen besitzen ebenso ein Eigenleben, das es ihnen ermöglicht, auch dann vorzukommen, wenn die entsprechende Wahrnehmung nicht mehr stattfindet. Dieses von der Sinnlichkeit losgelöste Vorstellungsleben drückt sich nicht nur in Fantasie und Traum aus, sondern ist auch Bedingung für die strebende und bisweilen willentliche Lenkung des Verhaltens. Ohne solchen Anhalt wäre das Verhalten nicht nur blind, sondern auch unvermögend, Handlungen zu initiieren. Durch Vorstellungen kann der Leib aktiv werden, auch wenn es keine auslösenden sinnlichen Reize gibt. Das Vorstellungsleben stellt den pathischen Raum dar, in dem Gefühle erweckt, gehemmt und verschoben werden.

18 De anima 435 b.

19 Wenn Aristoteles im zweiten Buch der Rhetorik verschiedene Gefühle analysiert, geschieht es in diesem Kontext: der Redner soll seinen Zuhörern zeigen, dass alles nicht gleich wichtig ist, d.h. auf die Zuhörer einwirken und sie von der Wichtigkeit seiner Sache überzeugen. 
Wenn es ein Eigenleben der Vorstellungen gibt, existieren ihre Gehalte auch in anderen Akten als die in enger Hinsicht sinnlichen und werden von dem konkreten sinnlichen Hintergrund unabhängig - in andersartiger Aktualisierung auch neue Konnotationen aufnehmen können. Träume sind nur ein extremes Beispiel dafür, aber die Phantasmagorie ist keineswegs nur ein oneirisches Phänomen, sondern stellt vielmehr ein Medium der Bearbeitung von Möglichkeiten jenseits der sinnlich vermittelten Wahrnehmung dar. Die dramatische Bedeutung des Wortes ,Vorstellung“ scheint hier fast wichtiger zu sein als die mit der Repräsentationsannahme verbundene Idee einer mentalen Vertretung. Inhalte ereignen sich in einem bedeutsamen Kontext und üben aufeinander Einfluss aus, bieten der betroffenen Person zugleich ein reiches Feld von potentiell handlungsauslösendem Sinn, in dem mit Möglichkeiten gespielt werden kann, ohne sie unumkehrbar zu verwirklichen.

Im intentional perzipierenden Vorgang entstehen die anhaltsverleihenden Vorstellungen (die intentionalen Gehalte) im Rahmen der Gerichtetheit des Lebewesens, d. h. sind von dem Erstrebten (orekton) bedingt und deshalb eher responsiv oder reaktiv als rein spontan. Im Wechselspiel von aisthêsis und orexis werden strebensbedingte, emotiv gefärbte Dinge in der Welt wahrgenommen, was sich auch in das Gedächtnis sedimentiert. ${ }^{20} \mathrm{Ob}$ man deswegen die thomistische Auffassung übernehmen muss, dass alles, was im Verstand ist, vorher in der Sinnlichkeit war (nihil in intellectum quod non fuerit prior in sensu), kann hier offen bleiben. Die Frage ist, wie eigenständig das von der Sinnlichkeit losgelöste Vorstellungsleben ist. Wenn hier nur eine mnemische Transformation der sinnlich einheitsstiftenden phantasmata vermutet wird, scheint die emotive Kraft dieser Funktionen unterschätzt zu werden, die sowohl in der Sinnlichkeit wirkt wie auch von der Sinnlichkeit unabhängig weiterwirken kann. Vieles spricht dafür, dass es für das Selbstverständnis des Menschen entscheidend ist, gerade diese real oder potentiell bewegende Emotionalität zu verstehen - was vor allem in der responsiven Apperzeption geschieht.

\section{Die Sinne und der Sinn}

Viel ist darüber geschrieben worden, was der Gemeinsinn (koinê aisthêsis) bei Aristoteles letztendlich bedeutet. Die Interpretation wird dadurch nicht einfacher, dass der Ausdruck später Geschichte gemacht hat, u. a. als sensus communis und common sense. Von den Einzelsinnen scheint der Gemeinsinn

20 Henri Bergson nennt diese Funktion des Gedächtnisses souvenir pur. Henri Bergson, Matière et mémoire, Paris: PUF, 1990 [1939]. 
unterschieden zu sein, aber bei Aristoteles heißt dies sicher nicht, dass er z. B. mit ,gesundem Menschenverstand' gleichgesetzt werden kann. Eher scheint der Gemeinsinn etwas mit dem zu tun zu haben, was wir auf Deutsch meinen, wenn wir sagen, dass wir etwas ,im Sinn haben'. Wir beabsichtigen dann etwas, d. h. wir drücken eine Richtung im Denken und Handeln aus. Sinn kann eben auch Richtung bedeuten, und dies lässt sich aristotelisch besonders gut verstehen, insofern die Seele für Aristoteles die erste Gerichtetheit des Lebewesens darstellt. Der Gemeinsinn wäre dann mit orexis verknüpft - was die Deutung unterstützt, dass aisthêsis generell mit orexis verbunden ist. Vielleicht kann man koinê aisthêsis auch mit ,Gemüt' übersetzen. Wenn Aristoteles in der Rhetorik analysiert, wie Pathos vom Redner heraufbeschworen werden kann, geht es gerade darum, die Gemüter zu erregen. Dieser emotionale Einfluss ist deshalb so wichtig, weil die Gemütslage auch einen direkten Einfluss darauf hat, wie die Zuhörer etwas sehen, wie sie empfinden und wahrnehmen. Dieser Einfluss ist jedoch nicht so stark, dass der Zuhörer nach einer Rede rot als grün, Lärm als Melodie, Gestank als Duft wahrnehmen würde. Die Sinne haben eine gewisse Eigenständigkeit, und der Unterschied zwischen den verschiedenen Sinnesvermögen und dem Gemeinsinn bleibt auch deshalb bestehen. Aristoteles hat eine durchaus realistische Konzeption der sinnlichen Qualitäten und geht davon aus, dass diese wirklich sind, weil sie schon vor dem Wahrnehmungsvorgang als wahrnehmbare Qualitäten vorlagen. Diese Qualitäten können aber nur dann in der Erfahrung aktualisiert werden, wenn ein Lebewesen durch emotionale Gemütslagen reizbar ist. Vielleicht könnte dies auf folgende Weise zusammengefasst werden: Pathos steht für emotive Intensität, die als solche keine Richtung hat, aber im Gemüt einen Sinn erhält, weil die Person ein strebendes Wesen ist. Das Leben dieser Person ereignet sich jedoch in einer Welt, in der es vieles zu berücksichtigen gibt, was schlichtweg nur gegeben ist. Ohne aisthêsis wäre die orexis ein blindes Beharren auf das bloße Vorankommen in eine gewisse Richtung und folglich zum Untergang verurteilt. Empeiria ist gerade aus diesem Grund so wichtig. Wie sich die Konturen der Welt in einer solchen Empirie abzeichnen, hängt von mehreren Funktionen ab: von den Einzelsinnen, vom Gemeinsinn (Gemüt), vom einheitsstiftenden Vermögen der Vorstellungskraft, von der Erregtheit und Reizbarkeit (Pathos) und generell von der strebenden Natur jenes Lebewesens, das lebendig gerade durch seine tragende Zweckgerichtetheit ist.

Wenn der Begriff des Empirischen in den letzten Jahrhunderten eine andere, spezifisch epistemische Bedeutung bekommen hat, ist dies vom besonderen Anliegen der neuzeitlichen Wissenskultur bedingt. Der gemeinsame Nenner dieser Verschiebung drückt sich als eine besondere Eingrenzung des sinnlichen Anlasses aus. Die sinnlichen Qualitäten werden dabei entweder 
als äußerliche Daten oder als Sekundärqualitäten verstanden - wobei nur die Ersteren von epistemischem Belang sind. Epistemisch irrelevant ist der ganze emotionale Bereich, der häufig als eine fast schattenhafte Dimension sonderbarer Personalität angesehen wird - eine Sichtweise, welche die Eingebundenheit des neuzeitlichen Subjekts in sein epistemisches Verhalten reduzieren soll. ${ }^{21}$ Was diesem Unterfangen als Ideal vorschwebt, ist ein bloßgelegter Objektbereich, der vom Pathischen und generell Seelischen gereinigt ist. Der Zugang soll gerade nicht durch Teilhaftigkeit an und in der Wirklichkeit gewonnen werden, sondern umgekehrt, durch die Situierung des Erkennenden in einer durch methodische Askese gewonnenen ,exzentrischen' Position, die den epistemisch streng differenzierenden Blick des neuen unbeseelten Bewusstseins ermöglicht.

Das Paradoxale an diesem Ideal besteht darin, dass es mit einer besonders starken Hervorhebung der Erkenntniszwecke einhergeht und folglich ein epistemisches Streben voraussetzt, dessen Folgen und Effizienz gar nicht zu bezweifeln sind. Wer auf diese Weise strebt, bleibt ein orektisches, von seinen Vorstellungen und seinem Gemüt getriebenes Wesen, läuft aber - wenn die subjektivierte Erkenntnisteleologie dominant wird - Gefahr, sich der Möglichkeit zu verschließen, dieses Getriebensein auch zu verstehen. Was zunächst als ein Erkenntnisgewinn erscheinen kann, zeigt sich dann als Verständnisverlust und bringt uns zurück zu den realen Bedingungen der Empirie, d. h. zu den vorherbestimmenden Dispositionen und zu derjenigen Anwesenheit, die es diesen Dispositionen ermöglicht, auch wirksam zu werden. Auf dieser Ebene der ,strukturierten Präsenz' ist Zugehörigkeit und Teilhabe, nicht exzentrische Positionalität das Entscheidende. Die Anwesenheit lässt etwas erscheinen, wovon wir abhängig sind, aber das wir trotzdem nicht nach Belieben handhaben können. Man könnte die Anwesenheit als solche mit Boethius das stehende Jetzt (nunc stans) nennen, ${ }^{22}$ eine als reine Gegenwart

21 Es kann hier auf eine seltsame Zweideutigkeit im Subjektbegriff hingewiesen werden: einerseits wird das Subjekt im Sinne der in der Neuzeit dominierenden Subjektphilosophie als archimedischer Punkt der Erkenntnis verstanden (Descartes, Kant u. a.), andererseits hat ,das Subjektive' im gängigen Sprachgebrauch immer mehr die Bedeutung des persönlich Ephemeren bekommen. Es ist schwierig zu sehen, wie sich das epistemisch maßgebende Subjekt der Theorie mit der heute gängigen Auffassung des Subjektiven vereinbaren ließe. Das Spannungsverhältnis erweckt vielmehr den Eindruck eines im Hintergrund tatsächlich vorliegenden Problems, zu dessen Lösung ein genaueres Verständnis der Beziehungen zwischen Affektivität und Erkenntnis dringend nötig ist.

22 Boethius, De trinitate I \& De consolationae philosophiae V. Die Gegenwart ist enigmatisch, weil sie einerseits ein Bestandteil der fortlaufenden Zeit ist, andererseits aber auch die Instanz darstellt, die als pure Anwesenheit etwas Außer- oder Überzeitliches manifestiert, das häufig als Beständigkeit oder Ewigkeit verstanden wurde. In der christlichen Tradition 
verstandene Unbestimmtheit, die auch zu unserer Verständnisfähigkeit beiträgt. Weil Aktualität mit dem zeitlichen Fortgang der Erfahrung verknüpft bleibt, ist dasselbe Jetzt jedoch auch ein laufendes (nunc currens), ein Glied in der Verwirklichung des Vorherbestimmten. Aktualität kann in dieser Hinsicht auch eine gute Gelegenheit (kairos) sein, um etwas zu tun, aber grundsätzlich ist Präsenz etwas anderes: die aktualisierende Grundbedingung der zeitlichen Vorgänge, die - wenn sie responsiv wird - auch zur menschlichen Seinserhellung beitragen kann.

\section{Bibliographie}

Alfred Jules Ayer, The Foundations of Empirical Knowledge, London: Macmillan, 1963 [1940].

Henri Bergson, Matière et mémoire, Paris: PUF, 1990 [1939].

Maine de Biran, Influence de l'habitude sur la faculté de penser, in: ders., OEuvres. Hg. von François Azouvi, 13 Bde., Paris: Vrin, Bd. 2 (1987): Mémoires sur l'influence de l'habitude. Hg. von Gilbert Romeyer-Dherbey, S. 124-336.

Otto Bollnow, Das Wesen der Stimmungen, Würzburg: Königshausen \& Neumann, 2009 [1941].

Otto Bollnow, Lebensphilosophie und Existenzphilosophie, Würzburg: Königshausen \& Neumann, 2009.

Martin Buber, Ich und Du, Stuttgart: Reclam, 1995 [1923].

Étienne Bonnot de Condillac, Traité des sensations, Paris: Fayard, 1984 [1754].

Wilhelm Dilthey, Grundlegung der Wissenschaften vom Menschen, der Gesellschaft und der Geschichte, in: ders., Gesammelte Schriften. Hg. von Helmut Johach und Frithjof Rodi, 26 Bde., Göttingen: Vandenhoeck \& Ruprecht, Bd. 19 (1982).

Martin Heidegger, Die Grundbegriffe der Metaphysik. Welt - Endlichkeit - Einsamkeit, Frankfurt a. M.: Klostermann, 1983 [Vorlesungen 1929-30].

Martin Heidegger, Sein und Zeit, Tübingen: Max Niemeyer Verlag, 1986 [1927].

Martin Heidegger, Der Ursprung des Kunstwerkes, Stuttgart: Reclam, 1988 [1950].

war gerade die aeternitas etwas Göttliches und wurde auch bei Boethius von der endlosen (laufenden) sempiternitas unterschieden. Die Bedeutung dieser Idee für Theologie, Kunst und Philosophie kann gar nicht überschätzt werden. Das stehende Jetzt ist kein Anhalt der weiterwollenden Erfahrung, sondern vielmehr der innerzeitliche Ausdruck der Parusie - was uns nicht als Gehalt und Anhalt dient, sondern uns stattdessen in unserem Streben anhält. Wie sich das stehende und das laufende Jetzt zu einander verhalten, ist ein Zankapfel der Tradition. Aristotelisch gedacht, scheint mir die Verbindung das Wesentliche zu sein. 
Edmund Husserl, Erfahrung und Urteil. Untersuchungen zur Genealogie der Logik, Hamburg: Meiner, 1999.

Jan-Ivar Lindén, „Intentionnalité et perception: une esquisse aristotélicienne“, in: Chôra (2011 / 2012).

Jan-Ivar Lindén (Hg.), Aristotle on Logic and Nature, Leuven: Peeters, 2019.

Perceiving, Sensing and Knowing. Hg. von Robert J. Swartz, New York: Doubleday, 1965. Ludwig Wittgenstein, Philosophische Untersuchungen, Frankfurt a. M.: Suhrkamp, 1980 [1953]. 